\title{
High-Quality Input Choice under Uncertainty and Ambiguity: An Exploratory Study of Costa Rica's Coffee Sector
}

\author{
Joselyne Najera, Paula Arzadun, Monica Navarro, Martin Solis \\ School of Business Administration, Instituto Tecnológico de Costa Rica (TEC) \\ jnajera@daad-alumni.de,parzadun@itcr.ac.cr, monavarro@itcr.ac.cr, martin12cr@yahoo.com
}

\begin{abstract}
The purpose of the present study was to analyze the effect of multiple variables on the decision to invest in high versus regular-quality coffee production inputs. Thereby, a laboratory experiment was conducted with one hundred twenty-three undergraduate students, and posterior logistic regressions with random intercept were executed to analyze the collected data. The results showed that when there is a difference in the investment cost between a coffee of higher quality and a coffee of lower quality (regular), there is a slight increase in the odds ratio of investment in quality coffee, when going from an uncertainty condition of income to one with certainty in income of a higher quality coffee. On the other hand, when the cost is equal for both types of coffee, there is a strong increase in the odds ratio when going from an uncertainty condition to one with certainty. In addition, it was found that both the possibility of loss if there is an investment in a higher quality coffee and the ambiguity in the probability of facing a favorable business climate, reduce the odds ratio of investing in higher-quality coffee.
\end{abstract}

Keywords: Coffee, input investment, uncertainty, loss aversion, income ambiguity.

\section{Introduction}

The maximization of agricultural production efficiencies has long been studied from different approaches, including the management of nutrients, diseases or soil (Huettel, Narayana, \& Odening, 2011; Mulebeke, Kironchi, \& Tenywa, 2015). The former has been a particular concern for economies that reply to their welfare and global transactions on agricultural activities (Welbaum, Sturz, Dong, \& Nowak, 2004). Regarding tropical agriculture, coffee is the most extensively traded commodity in the world (Whelan \& Newsom, 2014). In Costa Rica, coffee has been an integral part of its culture, politics, and economics for more than two centuries, and thereby it functions as a structural backbone to its rural economy (Díaz, 2015). Costa Rica's coffee production predominantly operates through small and medium-sized farms, with intensive practices concerning fertilizers, pesticides, higher-yield varieties and high-density planting (Courville, 2003). Following this line, the sector is regulated by the government via its semi-autonomous Coffee Institute (Icafe), which protects the quality and reputation of Costa Rica's coffee (Snider, Gutiérrez, Sibelet, \& Faure, 2017). However, according to Díaz (2015) most small and medium-sized farms tend to focus on the production of regularquality coffee instead of high-quality coffee, since the majority of Costa Rica's production comes from organizations that blend and sell different coffee quality varieties at a single price, usually based on the international stock market.

Such a predominant system opposes to the differentiated-receptacle system, which, under fixed contract conditions, offers an economic incentive for both regular- and high-quality production (Lewin, Giovannucci, \& Varangis, 2004). The aforementioned, is gaining importance as coffee prices set at the New York Board of Trade have fallen amid a rise in the overproduction of coffee of less quality (e.g., robust variety) and its consequent blending with higher quality coffee (Jaffee, 2014). In order to identify key elements that influence the decision to invest more money in the production of higher-quality coffee versus regular-quality coffee, the present study analyzed the effect of the following variables in the decision-making process: a) income ambiguity associated with producing higher-quality coffee; b) equalization of the production costs between higher-quality coffee and regular-quality coffee; c) money loss probability due to investing in higher-quality coffee; and d) favorable versus unfavorable business climate ambiguity. The present investigation provides new evidence to Costa Rica's coffee production sector, which can contribute to increase high-quality coffee production, through the improvement of incentives that take into consideration current production and market constraints (Saenger, Qaim, Torero, \& Viceisza, 2013). 


\section{Literature Review}

Based on the above, we conducted a previous exploratory qualitative study with small and medium-sized Costa Rican coffee producers. After a series of in-depth interviews, we found potential biases that can influence the decision to invest in regular-quality coffee, instead of high-quality coffee. Below, we describe such biases and then explain how they relate to product decisions. Especially, since there is scarce empirical evidence concerning proper contract design in the small farm context (Bellemare, 2010; Hueth, Ligon, Wolf, \& $\mathrm{Wu}, 1999$; Saenger, Qaim, Torero, \& Viceisza, 2013)

Status Quo and Loss Aversion: During decision-making, there are a number of elements that can condition an election of choice. According to Samuelson and Zeckhauser (1988), for instance a person can either decide to make no decision, make a new decision, or keep the original decision. However, according to the authors, when people are faced with new alternatives, they tend to persist on maintaining the old ones as a course of action, due to the influence of factors, such as the fear of losing status quo by taking a radical decision. Following this line, Samuelson and Zeckhauser (1988) explain the above according to three categories: rational decision-making, erroneous cognitive perceptions and psychological commitment. Regarding rational decision, the status quo bias can occur in two situations: 1) when the costs of making a change outweigh the benefits (i.e., transition costs); 2) when facing uncertainty. In this case, the option that is already known to be reliable is maintained. Concerning the erroneous cognitive perceptions, there are also two alternatives: 1) when these perceptions are linked to a feeling of loss or gain, 2) when new decisions are made based on the initial decisions. Finally, psychological commitment refers to the desire to firmly hold an initial decision. At a scientific level, it is argued that the status quo bias is based on the bias of aversion to loss, because the status quo functions as an initial reference point, from which the extra benefits on the current situation are considered (Eidelman \& Crandall, 2012; Kahneman, Knetsch, \& Thaler, 1991; Anderson, 2003). Tversky and Kahneman (1991) point out the loss aversion as the perception that wins are lower than losses. People prefer to avoid the suffering of a loss than the benefits of a gain.

An aspect that is highlighted in the study by Tversky and Kahneman (1991) is that when a subject must define what they are willing to receive for delivering an asset that he/she evaluates the possibility of losing because of obtaining less than expected. This bias can be present when the farmer faces the decision to change the production from regular coffee to high-quality coffee. In this sense, we found that the interviewed coffee producers shared a concern over the possibility of losing money in the process amid a possible increase in their investment, since the production of higher quality coffee implies a bigger investment on input quality and wages. Because of this finding, we evaluated if the possibility of losing money in a scenario of coffee inversion influence the decision to produce regular coffee instead of high-quality coffee that might provide more gains. Following this line, we determined that such loss aversion bias could be reduced if the coffee cooperative provides a credit that incentivizes the production of higher quality coffee in its small and medium-sized associates. As sustained by Tversky and Kahneman (1991), people adapt to gains or losses depending on their reference points; hence, a monetary aid could provide a more promising expected future state (Van Raaij, 2016). In this way we assessed in a scenario of coffee inversion if a credit given to reduce the increasing cost in the production of high-quality coffee that is discounted from de earning of the coffee production, incentives the inversion in high-quality coffee. Hence, we expected that the credit would hinder the negative effect of loss aversion.

Ambiguity Bias: People tend to dislike the uncertainty more than the risk; therefore, they prefer not to bet when they do not know the probability of occurrence of an event (Pompian, 2006). This was discovered in a simple game carried out by Ellsberg (1961). In an urn, he placed 50 red balls and 50 black balls, while in another urn there were 100 balls that might be red or black, hence there was an unknown probability distribution for each player. Ellsberg found that under this environment the majority of the people prefer to bet when in the first urn, when they know the probability distribution. The results obtained by Ellsberg have been confirmed in different population and regions, for example with non-student subjects (Butler, Guiso, \& Jappelli, 2014; Dimmock, Kouwenberg, Mitchell, \& Peijnenburg, 2013; Dimmock, Kouwenberg, \& Wakker, 2015), with non-Western subjects (Akay, Martinsson, Medhin, \& Trautmann, 2012; Engle-Warnick, Escobal, \& Laszlo, 2007), with children (Sutter, Kocher, Glätzle-Rützler, \& Trautmann, 2013), monkeys (Hayden, Heilbronner, \& Platt, 2010), etc. 
The ambiguity aversion is influenced by the context in which the decision is taken. For example, Fox and Weber (2002), found four aspects of context that can make ambiguity more attractive or less attractive. These aspects are as follows a) people find an ambiguous situation more attractive when it shown after a situation less familiar and on the contrary finds an ambiguous situation less attractive when a more familiar ambiguous situation has been shown previously. b) An ambiguous family situation is more attractive if it is exposed later to a less familiar situation, with respect to whether it is exposed first. c) An ambiguous situation becomes less attractive when you are exposed to diagnostic information that people do not know how to use. 4) In situations of competition, people who feel more competent see ambiguity as more attractive, however when there is no competition, the opposite occurs. In the qualitative interviewee we identified a possible ambiguity aversion bias since the interviewees were not sure about the total amount of retribution they were going to get if they produced higher quality coffee, which implied missing information that could inhibit the decision to invest in a higher quality coffee.

The ambiguity aversion bias alludes to people's tendency to consider options as less attractive when the probability of gaining is ambiguous (Ellsberg, 1961), since people are usually more uncomfortable with ambiguity than with risk (Pompian, 2006). Lastly, we determined that stochastic conditions could also affect the quantity-quality trade-off, since under malign conditions the output quality tends to vary due to a variation in the access to optimal production inputs (Saenger, Qaim, Torero, \& Viceisza, 2013). In this line, the data showed that Costa Rica's business climate represents a stochastic component that can affect the potential outcome of coffee production since for instance an elevated inflation rate or limited economic incentives can hinder the investment on high-quality production inputs and technology. Due to the above conditions, we evaluate through a coffee investment game if the ambiguity in the income of the highest quality coffee and the ambiguity in the probity of the business climate influence the decision to invest in higher quality coffee.

\section{Method}

Experimental Design: Participants assumed the role of a coffee producer who has 1500 ECUs available to invest in the production of the crop. They had to decide to invest that amount in either producing type "A" (higher- quality) or type "B" (regular-quality) coffee. Each type of coffee had a specific investment cost and a sales income that varied depending on favorable or unfavorable business climate. Both business climates were associated with a probability of occurrence. Each subject obtained a profit from the experiment that depended on the following formula: $1 \mathrm{ECU}=1500$ - Investment cost + sales income. The exchange rate of ECUs to Costa Rican colons was of $\not 1.9 /$ ECU. The average payment was $\$ 7.9$ per subject. The lowest earner received $\$ 3.3$, and the highest earner received $\$ 11.9$. The experiment had different treatments, using a mixed-design. Therefore, it had both a between-subjects and within-subjects design. The treatments between subjects derived from a $2 * 2$ factorial design.

\section{The factors were:}

1) The difference in costs between types of coffee. It had two levels: a) costs differentiated by type of coffee and b) same costs. This equalization in costs represented a temporary subsidy, in which the investment cost in coffee "A" was reduced. However, the reduced amount was deducted at the end from the sales income.

2) Ambiguity in the sales' income of the highest quality product. It had two levels: a) with ambiguity and b) without ambiguity. Ambiguity occurred when a range of income was provided instead of a specific amount. With the combination of these two factors, the experiment was set with four treatments, as detailed in table 1.

Table 1: Distribution of the Treatment between Groups.

\begin{tabular}{lll}
\hline \multirow{2}{*}{ Factor Costs } & Factor income ambiguity & \\
\cline { 2 - 3 } & With ambiguity & Without ambiguity \\
\hline Differentiated & T0 & T2 \\
Equal (temporal subsidy) & T1 & T3 \\
\hline $\begin{array}{l}\text { Notes: T0stands for treatment group 1; T1 for treatment group 2; T2 for treatment group 3; T3 for treatment } \\
\text { group 4. }\end{array}$
\end{tabular}


The participants were randomly assigned to different treatments as follows: 31 in the treatment T1, 29 in the treatment T2, 31 in the treatment T3, and 32 in the treatment T4. Each subject had to face four rounds, which represented different scenarios. The scenarios also derived from combining two factors, of two levels each:

- Ambiguity in the probability of the business climate that will occur. It had two levels: a) without ambiguity; and b) with ambiguity. When there was no ambiguity, the probability of facing a favorable climate consisted of 0.60 . Otherwise, it was not indicated and a random probability between 0 and 1 was randomly generated for payment purposes.

- The possibility of loss when investing in the higher quality product when the weather is unfavorable. It had two levels: a) with no possibility of loss; and b) with the possibility of loss. When there was a possibility of loss, the investment cost could be higher than the sales income, if an unfavorable climate occurred. The combination of these factors defined the four scenarios, as detailed in table 2. As those scenarios were within-subjects the order in which each player faced them was randomized.

Table 2: Scenarios in Each Treatment

\begin{tabular}{lll}
\hline \multirow{2}{*}{$\begin{array}{l}\text { Ambiguity in probability of business } \\
\text { climate }\end{array}$} & Possibility of loss & \\
\cline { 2 - 3 } & With no possibility & With possibility \\
\hline Without ambiguity & $\mathrm{S} 1$ & $\mathrm{~S} 2$ \\
With ambiguity & $\mathrm{S} 3$ & $\mathrm{~S} 4$ \\
\hline
\end{tabular}

Notes: S1 stands for scenario number one; S2 for scenario number two; S3 for scenario number three; S4 for scenario number four.

In the appendix, we present the investment cost and income values according to the business climate assumed by each treatment, between subjects for the four scenarios. Regardless of the treatment, in scenarios S1 and S2, the difference in the expected payment between coffee "A" and coffee "B" was of 200 ECUs, while in scenarios S3 and S4 the difference consisted of 100 ECUs, assuming a probability of 0.5 . In this way, in any treatment, there was a higher income for coffee "A". In terms of the data analysis approach, two logistic linear regression models were executed for each scenario to test the effects of ambiguity in sales and differential cost, using the selection of coffee "A" against coffee "B" as the dependent variable. In the first model, the variables ambiguity in sales for coffee " $\mathrm{A}$ " and differential cost were added. In the second model, the variables mentioned and the interaction between them were included. Finally, to test the effects of ambiguity in the probability of a specific business climate and the possibility of losing, two mixed logistic models with a random intercept were executed, using the selection of coffee "A" against coffee " $B$ " as the dependent variable. These models were applied as individuals were nested in rounds (scenarios), which may have affected the independence of residuals.

Participants: The experiment was conducted during October 2017, at the Experimental Economics Lab (LEXTEC) from the Costa Rica Institute of Technology (TEC). The experiment was programmed with the computer-programming language Java. A total of one hundred twenty-three undergraduate students participated in the experiment, from which forty-one percent were women and fifty-nine percent were men, with an average age of 21.4 years (ages ranging from 18 to 32 years). The sample size was estimated according to the budget available. The students were distributed among seven sessions, lasting approximately 15 minutes. The recruitment was possible through LEXTEC's own SR 1.0 system, which allowed the participants to register and consequently participate in the study. The researchers made sure that all participants had never been on one of LEXTEC's previous experiments, and that each one read and signed an informed consent before the experimental session. We use students because of the difficulty to find farmers available in the harvest time and because the cost of participating in an experiment for a student is certainly lower (Fréchette, 2011) it is a common practice in laboratory experimental economics use students as subjects (Danielson \& Holm, 2007). Besides, student and nonstudent subjects have shown similar behavior in the laboratory in different research (Charness \& Villeval, 2009; Güth \& Kirchkamp, 2012; Güth, Schmidt, \& Sutter, 2007; Alm, Bloomquist, \& McKee, 2015). 
Analysis Method: Logistic regression models were generated to analyze the effect of income ambiguity, differentiated cost and the interaction between these variables on the decision of inversion in each scenario. Logistic regression is used because the decision of inversion is composed of two options, high-quality coffee (coffee A) or regular-quality (coffee B). Finally, to analyze the effects of the possibility of loss and ambiguity in the probability of facing a favorable climate, as well as the interaction between these variables, two logistic regression models were generated with random intercept. A multilevel model was used because all the experimental subjects faced the scenarios from combining these two variables. In this way, there was a correlation between the residuals of the model that must be considered, before estimating the standard errors of the coefficients. Ignoring this situation by estimating classic logistic regressions would lead to the analysis of biased standard errors and therefore to flawed conclusions about the significance level of the coefficients (Snijders \& Bosker, 1999).

\section{Results}

Figure 1 contains the percent of subjects who chose coffee high-quality (coffee " $\mathrm{A}$ ") by treatment and scenario. It shows that the percentage of each treatment varies through each scenario considerably. Moreover, there are scenarios where a single treatment is the most effective, while in others it is the least effective. For example, T1 has the highest percentage in S1, but the lowest in S3.

Figure 1: Percentage of Subjects Choosing Option a, by Treatment (T) According to Each Scenario (S).

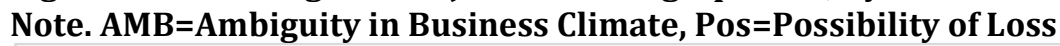

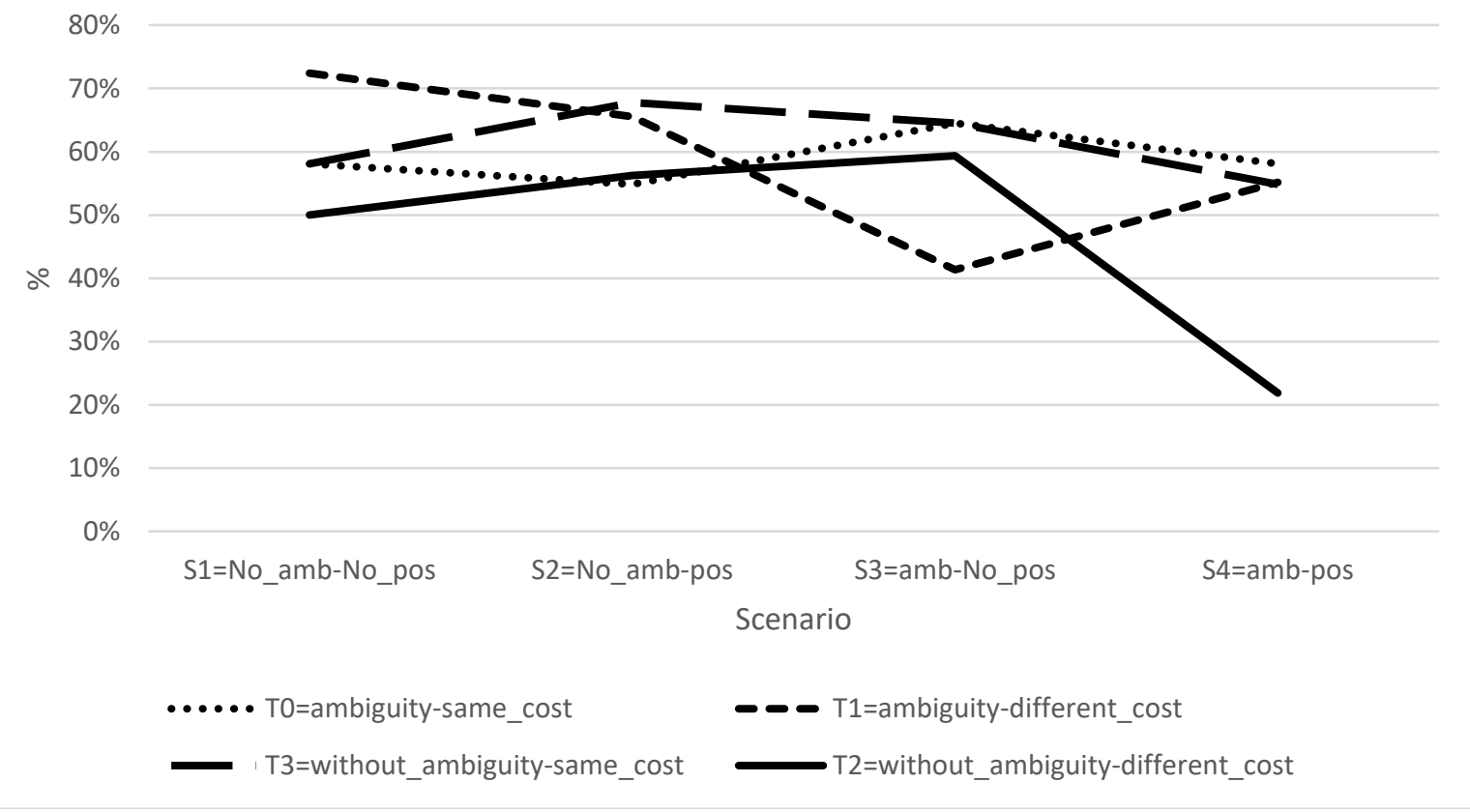

Among the most relevant results, the highest investment in high-quality coffee (coffee A), approximately $72 \%$, happened on S1 (probability of favorable climate known, and without a possibility of loss) and T1 (equal costs and ambiguity in income). The lowest investment approximately 22\%, occurred on S4 (probability of known favorable climate, and a possibility of known loss) and T3 (same costs and without ambiguity in income). These results suggest that in the most unfavorable conditions the equality of costs has an adverse effect if the income is known with certainty. However, under favorable conditions, the equality of costs generates the most positive effect, if there is ambiguity in income. The analysis of data by treatment provides a general perspective of the results obtained. However, it does not allow identifying variables or effects that significantly influence the choice of high-quality coffee (coffee A) over regular-quality (coffee B). Additionally, logistic regression models were generated, with the results detailed in Table 3 and Table 4. Table 3 shows the effect of income ambiguity and cost equality on the choice of option A. 
For each scenario, two models were generated. In the first one, the simple effects were analyzed, while in the second, the interaction between income ambiguity and cost equality was examined. The results indicate that only in S4 - where the conditions were more unfavorable due to a possibility of loss in the case of coffee "A" and ambiguity in the probability of a favorable climate - the two variables exerted a significant influence on the choice of coffee "A". Specifically, in S4 there was an effect of interaction between the two variables.

Table 3: Logistic Regression Coefficients of Ambiguity and Cost Effects on an Option Choice

\begin{tabular}{|c|c|c|c|c|c|c|c|c|}
\hline \multirow{2}{*}{ Effects } & \multicolumn{2}{|c|}{ Scenario 1} & \multicolumn{2}{|c|}{ Scenario 2} & \multicolumn{2}{|c|}{ Scenario 3} & \multicolumn{2}{|c|}{ Scenario 4} \\
\hline & Model 1 & Model 2 & Model 3 & Model 4 & Model 5 & Model 6 & Model 7 & Model 8 \\
\hline (Intercept) & 0.09 & 0.33 & 0.5 & $0.74^{*}$ & $0.79 *$ & -0.6 & -0.11 & 0.19 \\
\hline $\mathrm{AMB}$ & 0.46 & 0.00 & -0.08 & -0.55 & -0.37 & 0.00 & $0.76^{* *}$ & 0.13 \\
\hline Cost & 0.12 & -0.33 & -0.03 & -0.49 & -0.58 & -0.22 & $-0.77^{* *}$ & $-1.47^{* *}$ \\
\hline $\mathrm{AMB}^{*}$ cost & & 0.97 & & 0.94 & & -0.73 & & $1.35^{* *}$ \\
\hline
\end{tabular}

Notes: $\mathrm{AMB}=$ ambiguity in income (1=yes, $0=$ no); cost= same cost $(1=y e s, 0=$ no);

${ }^{*} \mathrm{p}<0.10,{ }^{* *} \mathrm{p}<0.05$

Figure 2 shows the effect of the interaction between ambiguity in income and difference in cost. In this sense, the data show that when there were differences in the cost there was a slight increase in the odds ratio of high-quality coffee (coffee A) when going from an unambiguous condition of income to one with ambiguity in income. On the other hand, when the costs were equal, there was a substantial increase in the odds ratio when going from an unambiguous condition to one with ambiguity. Even in the case of non-ambiguity, the equality of costs inhibited the choice of option A, while ambiguity propelled it positively.

Figure 2: Interactions between Ambiguity in Income and Difference in Cost on Natural Logarithm Odds Ratio of option "a" Choice

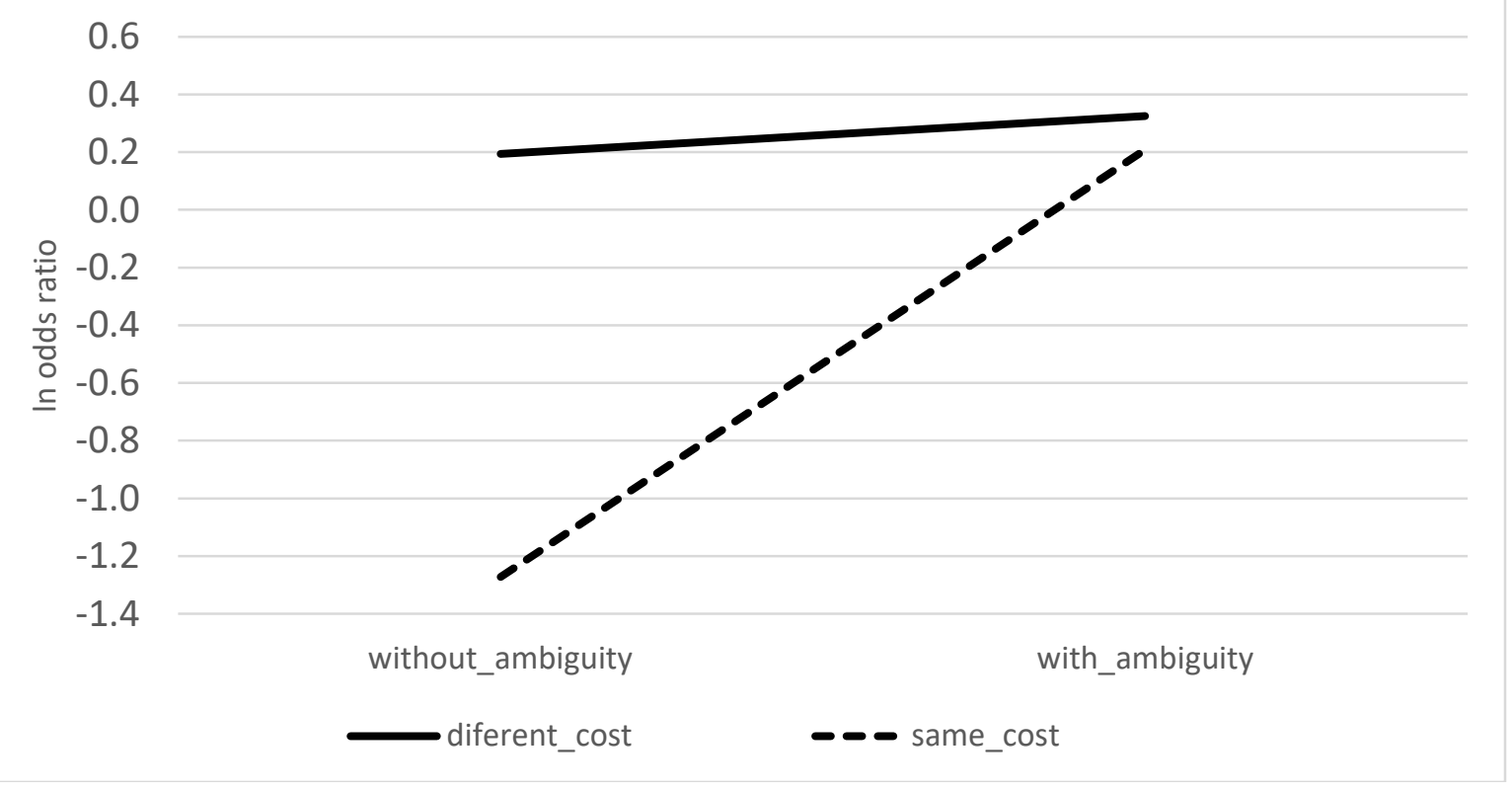

Finally, to analyze the effects of the possibility of loss and ambiguity in the probability of facing a favorable climate, as well as the interaction between these variables, two logistic regression models were generated with random intercept. A multilevel model was used because all the experimental subjects faced the scenarios from combining these two variables. In this way, there was a correlation between the residuals of the model that must be considered, before estimating the standard errors of the coefficients. Ignoring this situation by estimating classic logistic regressions would lead to the analysis of biased standard errors and therefore to flawed conclusions about the significance level of the coefficients (Snijders \& Bosker, 1999). 
Table 4: Logistic Regression Coefficients of Ambiguous Probability and Loss Possibility Effects on an Option Choice

\begin{tabular}{lll}
\hline Effects & Model 1 & Model 2 \\
\hline Fixed & & \\
(Intercept) & 0.22 & 0.22 \\
Position & 0.23 & 0.21 \\
Loss & $-0.42^{*}$ & -0.3 \\
AMB pro & $-0.62^{* *}$ & -0.5 \\
loss* AMB pro & & -0.18 \\
Random & 0.48 & 0.48 \\
(Intercept) & &
\end{tabular}

Table 4 shows the coefficients of the two models. In the first model, two simple effects were evaluated, while in the second, the interaction between the two was analyzed. It should be noted that in both models, the position or game round in which each effect appears is added as a control variable. In model 2, it was observed that there is no interaction between both effects, but there is a significant influence of the simple effects on the choice of high-quality coffee (coffee A). Specifically, it was found that the possibility of loss reduces the logarithm of the odds ratio by -0.42 , and the odds ratio in $34 \%$ (1-exponent of -0.42 ), while the ambiguity in the probability of facing a favorable climate reduces the logarithm of the odds ratio in -0.62 and the odds ratio to $46 \%$.

Discussion: In order to identify key elements that influence the decision to invest more money in the production of higher-quality coffee versus regular-quality coffee, the present study analyzed the effect of the following variables in the decision-making process: a) income ambiguity associated to producing higherquality coffee; b) equalization of the production costs between higher-quality coffee and regular-quality coffee; c) money loss probability due to investing in higher-quality coffee; and d) favorable versus unfavorable business climate ambiguity. In terms of the effect of income ambiguity and the equalization of costs on the decision to invest more on higher-quality coffee, the researchers launched the proposition that when there was a financing of the additional costs concerning the production of higher-quality coffee, there would be an increase in the investment on such production input (even though such financial aid had to be returned after profiting). The above, was based on the premise that people tend to adapt to gains or losses based on their reference points, hence a financial aid that equalizes the costs for both regular and higherquality coffee production, would imply a new perspective on gain instead of loss (Tversky \& Kahneman, 1991). Still, the results obtained show that the expected premise was not accomplished. In three of the four formulated scenarios, when combining the possibility of loss and ambiguity with the probability of facing a favorable business climate, cost equality did not reflect a significant difference on the decision to invest in higher-quality coffee.

Even more, in the scenario with the highest uncertainty (i.e., people were unaware of the possibility of facing a favorable business climate and of losing money by investing in higher-quality coffee), differentiated costs were preferred. Conversely, it was observed that when there was income ambiguity, the equalization of costs increased the decision to invest in higher-quality coffee. A possible explanation is that the equality of costs might operate as a defense mechanism that mitigates the uncertainty arising from the ambiguity of income, the possibility of loss, and the missing probability of occurrence of an unfavorable scenario. As a practical implication, this result suggests that cost equalization mechanisms could be furtherly assessed as a mechanism to reduce the fear of investment in scenarios of greater uncertainty. Another result was that the ambiguity in the probability of occurrence of a favorable business climate hurts the investment in higher quality coffee, which is justified by the ambiguity aversion that has been demonstrated since the pioneering study of Ellsberg (1961). In this study, they found that people usually consider options as less attractive when the probability of gaining is ambiguous. Therefore, people tend to prefer options with risk than options where there is ambiguity (Eichberger, Oechssler, \& Schnedler, 2015). Finally, there was also an adverse effect on investment in higher quality coffee due to the possibility of loss in the event of an unfavorable business 
climate. This effect is explained by the loss aversion bias, which has shown that subjects tend to give more weight to an eventual loss than to a possible gain (Tversky \& Kahneman, 1991). The main limitation of the present research was the external validity, mainly since it was carried out with undergraduate university students, amid a limited financial budget and access to experimental subjects in the coffee sector. Having addressed the problem of a specific population the study should be replicated with a sample matching the corresponding population of Costa Rican coffee producers.

In which case the form of application must also be modified since it is not convenient to use computers because of the level of education of the population. The mixed design used, between and within subjects, facilitated the evaluation of several effects, which is an advantage considering that it is a first exploratory study. However, it does not allow the deepening into specific relationships for example, the interaction between the possibility of loss and the equality of costs, or ambiguity in the business climate and cost equality, among others. Also, future studies could be aimed at the producer's reaction to scenarios where there are different sources of ambiguity in the investment decision of both regular coffee and higher quality coffee. The contributions of studies such as those of Eliaz and Ortoleva (2015), Eichberger et al. (2015) can be valuable in this type of problems.

\section{Conclusion and Recommendations}

In the academic context, this study is the first to assess several variables associated with cognitive biases that can influence the decision to invest in higher quality products. New studies could deepen the analysis of new variables or biases that influence investment decisions. Additionally, efforts to take laboratory experiments to field experiments should be made to strengthen the external validity of these findings. As a practical implication, these results suggest that, as much as possible, some information should be given of the possibility of a favorable/unfavorable scenario or business climate to encourage investment in higher quality coffee. In addition, if the farmers know that they can lose money if an unfavorable climate occurs when investing in higher quality coffee, the investment will be reduced.

\section{References}

Akay, A., Martinsson, P., Medhin, H. \& Trautmann, S. T. (2012). Attitudes toward uncertainty among the poor: an experiment in rural Ethiopia. Theory and Decision, 73(3), 453-464.

Alm, J., Bloomquist, K. M. \& McKee, M. (2015). On the external validity of laboratory tax compliance experiments. Economic Inquiry, 53(2), 1170-1186.

Anderson, C. J. (2003). The psychology of doing nothing: forms of decision avoidance result from reason and emotion. Psychological Bulletin, 129(1), 139-167.

Bellemare, M. F. (2010). Agricultural extension and imperfect supervision in contract farming: evidence from Madagascar. Agricultural Economics, 41(6), 507-517.

Brenner, L., Rottenstreich, Y., Sood, S. \& Bilgin, B. (2007). On the psychology of loss aversion: Possession, valence, and reversals of the endowment effect. Journal of Consumer Research, 34(3), 369-376.

Brink, A. G. \& Rankin, F. W. (2013). The effects of risk preference and loss aversion on individual behavior under bonus, penalty, and combined contract frames. Behavioral Research in Accounting, 25(2), 145170.

Butler, J. V., Guiso, L. \& Jappelli, T. (2014). The role of intuition and reasoning in driving aversion to risk and ambiguity. Theory and Decision, 77(4), 455-484.

Charness, G. \& Villeval, M. C. (2009). Cooperation and competition in intergenerational experiments in the field and the laboratory. American Economic Review, 99(3), 956-78.

Courville, S. (2003). Use of indicators to compare supply chains in the coffee industry. Greener Management International, 43, 93-105.

Danielson, A. J. \& Holm, H. J. (2007). Do you trust your brethren?: Eliciting trust attitudes and trust behavior in a Tanzanian congregation. Journal of Economic Behavior \& Organization, 62(2), 255-271.

Díaz, D. (2015). Costa Rica: How quality pays for coffee farmers. Zurich: Responsibility Investments AG.

Dimmock, S. G., Kouwenberg, R. \& Wakker, P. P. (2015). Ambiguity attitudes in a large representative sample. Management Science, 62(5), 1363-1380. 
Dimmock, S. G., Kouwenberg, R., Mitchell, O. S. \& Peijnenburg, K. (2013). Ambiguity attitudes and economic behavior. National Bureau of Economic Research.

Eichberger, J., Oechssler, J. \& Schnedler, W. (2015). How do subjects view multiple sources of ambiguity? Theory and Decision, 78(3), 339-356.

Eidelman, S. \& Crandall, C. S. (2012). Bias in Favor of the Status Quo. Social and Personality Psychology Compass, 6(3), 270-281.

Eliaz, K. \& Ortoleva, P. (2015). Multidimensional Ellsberg. Management Science, 62(8), 2179-2197.

Ellsberg, D. (1961). Risk, ambiguity, and the Savage axioms. The quarterly journal of economics, 75(4), 643669.

Engle-Warnick, J., Escobal, J. \& Laszlo, S. (2007). Ambiguity aversion as a predictor of technology choice: Experimental evidence from Peru. CIRANO - Scientific Publications 2007s-01.

Fox, C. R. \& Weber, M. (2002). Ambiguity aversion, comparative ignorance, and decision context. Organizational behavior and human decision processes, 88(1), 476-498.

Fréchette, G. R. (2011). Laboratory Experiments: Professionals Versus Students. In G. R. Fréchette, \& A. Schotter, Handbook of Experimental Economic Methodology (pp. 360-389).

Güth, W. \& Kirchkamp, 0. (2012). Will you accept without knowing what? The Yes-No game in the newspaper and in the lab. Experimental Economics, 15(4), 656-666.

Güth, W., Schmidt, C. \& Sutter, M. (2007). Bargaining outside the lab-a newspaper experiment of a three-person ultimatum game. The Economic Journal, 117(518), 449-469.

Hayden, B., Heilbronner, S. \& Platt, M. (2010). Ambiguity aversion in rhesus macaques. Frontiers in neuroscience, $4,166$.

Hueth, B., Ligon, E., Wolf, S. \& Wu, S. (1999). Incentive instruments in fruit and vegetable contracts: input control, monitoring, measuring, and price risk. Review of agricultural economics, 21(2), 374-389.

Huettel, S., Narayana, R. \& Odening, M. (2011). Measuring dynamic efficiency under uncertainty. Sieg-Working paper 10.

International Coffee Organization. (2018). Trades Statistics Tables. Retrieved from International Coffee Organization: http://www.ico.org/trade_statistics.asp

Jaffee, D. (2014). Brewing Justice: Fair trade coffee, sustainability, and survival. Oakland, California: University of California Press.

Kahneman, D., Knetsch, J. L. \& Thaler, R. (1991). The endowment effect, loss aversion, and status-quo bias. Journal of Economic Perspectives, 193-206.

Lewin, B., Giovannucci, D. \& Varangis, P. (2004). Coffee markets: new paradigms in global supply and demand. World Bank Agriculture and Rural Development Discussion Paper No. 3.

Mulebeke, R., Kironchi, G. \& Tenywa, M. M. (2015). Exploiting Cropping Management to Improve Agricultural Water Use Efficiency in the Drylands of Eastern Uganda. Sustainable Agriculture Research, 4(2), 5769.

Pompian, M. M. (2006). Behavioral Finance and Wealth Management: How to Build Optimal Portfolios That Account for Investor Biases. New Jersey: John Wiley \& Sons, Inc.

Saenger, C., Qaim, M., Torero, M. \& Viceisza, A. (2013). Contract farming and smallholder incentives to produce high quality: experimental evidence from the Vietnamese dairy sector. Agricultural Economics, 44(3), 297-308.

Samuelson, W. \& Zeckhauser, R. (1988). Status quo bias in decision making. Journal of Risk and Uncertainty, $1(1), 7-59$.

Snider, A., Gutiérrez, I., Sibelet, N. \& Faure, G. (2017). Small farmer cooperatives and voluntary coffee certifications: Rewarding progressive farmers of engendering widespread change in Costa Rica? Food Policy, 69, 231-242.

Snijders, T. \& Bosker, R. (1999). Multilevel Analysis: An introduction to basic and advanced multilevel modelling. London: SAGE Publications.

Sutter, M., Kocher, M. G., Glätzle-Rützler, D. \& Trautmann, S. T. (2013). Impatience and uncertainty: Experimental decisions predict adolescents' field behavior. American Economic Review, 103(1), 51031.

Tversky, A. \& Kahneman, D. (1991). Loss aversion in riskless choice: A reference-dependent model. The quarterly journal of economics, 106(4), 1039-1061.

Van Raaij, W. F. (2016). Understanding Consumer Financial Behavior: Money Management in an Age of Financial Illiteracy. England: Palgrave Macmillan. 
Welbaum, G. E., Sturz, A. V., Dong, Z. \& Nowak, J. (2004). Managing Soil Microorganisms to Improve Productivity of Agro-Ecosystems. Critical Reviews in Plant Sciences, 23(2), 175-193.

Whelan, T. \& Newsom, D. (2014). Sustainable coffee farming: Improving income and social conditions protecting water, soil and forests. New York: Rainforest Alliance. Retrieved from https://www.rainforest-alliance.org/sites/default/files/2016-08/sustainable-coffee-farmingreport.pdf

\begin{tabular}{|c|c|c|c|c|c|}
\hline \multicolumn{5}{|c|}{ Game values for $\mathrm{TO}$} & \multirow[b]{2}{*}{$\begin{array}{l}\text { Favorable } \\
\text { climate } \\
\text { probability }\end{array}$} \\
\hline Scenario & Coffee & Cost & Favorable income & Unfavorable Income & \\
\hline \multirow{2}{*}{1} & $\mathrm{~A}$ & 1500 & 3550 y 3050 & 2050 y 1550 & \multirow{2}{*}{0.6} \\
\hline & $\mathrm{B}$ & 1000 & 2350 y 1850 & 2050 y 1550 & \\
\hline \multirow{2}{*}{2} & A & 1500 & 3050 y 2550 & 1550 y 1050 & \multirow{2}{*}{0.6} \\
\hline & $\mathrm{B}$ & 1000 & 1850 y 1350 & 1550 y 1050 & \\
\hline \multirow{2}{*}{3} & $\mathrm{~A}$ & 1500 & 3550 y 3050 & 2050 y 1550 & \multirow{2}{*}{ unknown } \\
\hline & $\mathrm{B}$ & 1000 & 2350 y 1850 & 2050 y 1550 & \\
\hline \multirow{2}{*}{4} & $\mathrm{~A}$ & 1500 & 3050 y 2550 & 1550 y 1050 & \multirow{2}{*}{ unknown } \\
\hline & $\mathrm{B}$ & 1000 & 1850 y 1350 & 1550 y 1050 & \\
\hline \multicolumn{6}{|c|}{ Game values for $\mathrm{T} 1$} \\
\hline Scenario & Coffee & Cost & Favorable income & Unfavorable Income & $\begin{array}{l}\text { Favorable } \\
\text { climate } \\
\text { probability }\end{array}$ \\
\hline \multirow{2}{*}{1} & A & 1000 & 3050 y 2550 & 1550 y 1050 & \multirow{2}{*}{0.6} \\
\hline & $\mathrm{B}$ & 1000 & 2350 y 1850 & 2050 y 1550 & \\
\hline \multirow{2}{*}{2} & A & 1000 & 2550 y 2050 & 1050 y 550 & \multirow{2}{*}{0.6} \\
\hline & $\mathrm{B}$ & 1000 & 1850 y 1350 & 1550 y 1050 & \\
\hline \multirow{2}{*}{3} & $\mathrm{~A}$ & 1000 & 3050 y 2550 & 1550 y 1050 & \multirow{2}{*}{ unknown } \\
\hline & $\mathrm{B}$ & 1000 & 2350 y 1850 & 2050 y 1550 & \\
\hline \multirow{2}{*}{4} & $\mathrm{~A}$ & 1000 & 2550 y 2050 & 1050 y 550 & \multirow{2}{*}{ unknown } \\
\hline & $\mathrm{B}$ & 1000 & 1850 y 1350 & 1550 y 1050 & \\
\hline \multicolumn{6}{|c|}{ Game values for $\mathrm{T} 2$} \\
\hline Scenario & Coffee & Cost & Favorable income & Unfavorable Income & $\begin{array}{l}\text { Favorable } \\
\text { climate } \\
\text { probability }\end{array}$ \\
\hline 1 & A & 1500 & 3300 & 1800 & 0.6 \\
\hline
\end{tabular}




\begin{tabular}{|c|c|c|c|c|c|}
\hline & $\mathrm{B}$ & 1000 & 2100 & 1800 & \multirow{3}{*}{0.6} \\
\hline \multirow{2}{*}{2} & $\mathrm{~A}$ & 1500 & 2800 & 1300 & \\
\hline & $\mathrm{B}$ & 1000 & 1600 & 1300 & \\
\hline \multirow{2}{*}{3} & $\mathrm{~A}$ & 1500 & 3300 & 1800 & \multirow{2}{*}{ unknown } \\
\hline & $\mathrm{B}$ & 1000 & 2100 & 1800 & \\
\hline \multirow{2}{*}{4} & $\mathrm{~A}$ & 1500 & 2800 & 1300 & \multirow{2}{*}{ unknown } \\
\hline & $\mathrm{B}$ & 1000 & 1600 & 1300 & \\
\hline \multicolumn{6}{|c|}{ Game values for $\mathrm{T} 3$} \\
\hline Scenario & Coffee & Cost & Favorable income & Unfavorable Income & $\begin{array}{l}\text { Favorable } \\
\text { climate } \\
\text { probability }\end{array}$ \\
\hline \multirow{2}{*}{1} & $\mathrm{~A}$ & 1000 & 2800 & 1300 & \multirow{2}{*}{0.6} \\
\hline & $\mathrm{B}$ & 1000 & 2100 & 1800 & \\
\hline \multirow{2}{*}{2} & $\mathrm{~A}$ & 1000 & 2300 & 800 & \multirow{2}{*}{0.6} \\
\hline & $\mathrm{B}$ & 1000 & 1600 & 1300 & \\
\hline \multirow{2}{*}{3} & $\mathrm{~A}$ & 1000 & 2800 & 1300 & \multirow{2}{*}{ unknown } \\
\hline & $\mathrm{B}$ & 1000 & 2100 & 1800 & \\
\hline \multirow{2}{*}{4} & $\mathrm{~A}$ & 1000 & 2300 & 800 & \multirow{2}{*}{ unknown } \\
\hline & $\mathrm{B}$ & 1000 & 1600 & 1300 & \\
\hline
\end{tabular}

